\title{
Facsimile ferrets may aid cystic fibrosis research
}

Since the late 1990s, when Ian Wilmut and Keith Campbell cloned Dolly, the nowfamous Finn-Dorset sheep, the pantheon of mammals cloned by somatic cell nuclear transfer (SCNT) has expanded to include cows, mice, goats, pigs, rabbits, cats, mules, rats, horses, and dogs. Now, researchers report a new addition to the cloned mammal club: Mustela putorius furos, the domestic ferret.

Ferrets have great potential for research because of the striking similarity between human and ferret pulmonary physiology, which makes ferrets good models for studies of influenza and other respiratory diseases. John F. Engelhardt and Li Ziyi, researchers at the University of Iowa (Iowa City), and their research team became interested in ferrets as an outgrowth of their interest in cystic fibrosis (CF), a common inherited disease characterized by the accumulation of thick, sticky mucus in the lungs that blocks the airways and causes premature death. The striking physiological similarity between the ferret and human lung would make ferrets ideal candi- dates for the study of CF, for which at present there is no appropriate animal model. To use a ferret in this capacity, however, a CF ferret would be necessary. Unfortunately, no such ferret has yet been found.

That lack has not discouraged Engelhardt and his fellow researchers; they have proposed to create a CF ferret. As a first step Engelhardt's team set out to clone wild-type ferrets using SCNT. Although Engelhardt and Li had other cloning models to follow, they had to tailor cloning protocols specifically to ferrets. After failed cloning attempts using superovulated eggs, Engelhardt's team decided to try the procedure using naturally ovulated oocytes. Once they had harvested the oocytes, Engelhardt and his colleagues enucleated them and placed ferret somatic cells into the perivitelline space. They then fused the cells via an electric pulse.

The result was two viable female ferrets that have since been successfully bred ( Dev. Biol., May). The next step toward the greater goal of this research is to insert a defective copy of the cystic fibrosis transmembrane

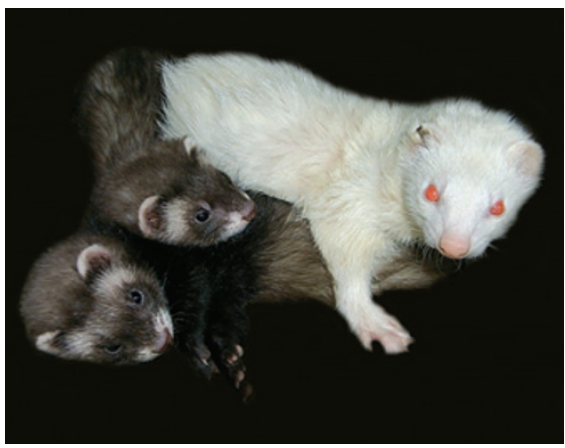

conductance regulator (CFTR) gene, which causes CF, into somatic ferret cells cultured in vitro. The nuclei from those cells can then be removed for use in cloning, eventually giving rise to a supply of ferrets that would be used for CF research.

How soon can we expect cloned ferrets for CF research? "Putting a time on getting a CF ferret is difficult," Engelhardt told Lab Animal, "we hope within 2 years, but the major variable involves the health and numbers of CFTR-targeted fibroblast cells we can obtain."

Owen Young

\section{NEW RHESUS MACAOUE_SOURCEITDENTIETED}

Nepali rhesus monkeys are genetically similar to animals native to India-good news for scientists faced with a continued shortage of these important research subjects.

For more than half a century, rhesus macaques (Macaca mulatta) of Indian origin have been the nonhuman primate (NHP) model of choice for biomedical and behavioral research. Studies using these monkeys have led to many medical advances, including the development of rabies and polio vaccines and the discovery of the Rh factor in blood. In recent years, the rhesus macaque's susceptibility to HIV-like viruses has made these animals an important model for studying HIV/AIDS. Due in part to India's 1978 ban on the exportation of NHPs for use in research, a scarcity of Indian macaques has led some researchers to opt for rhesus macaques of Chinese origin as substitutes, despite reports of genetic differences and variations in disease progression between animals from both countries.

Now, in a paper published in the June issue of the American Journal of Primatology, Randall C. Keyes of the University of Washington (Seattle) and colleagues suggest that Nepal-derived animals may be a better stand-in. The researchers collected blood from rhesus macaques living in Kathmandu, Nepal, and used the samples in mitochondrial and genomic DNA analyses. A comparison of the sequence results with those previously obtained from macaques of Indian and Chinese origin revealed that macaques of Nepali origin were more genetically similar to animals of Indian origin than to those from China. Keyes's group also looked for similarities in major histocompatibility complex (MHC) alleles, which have important implications for immune function and disease progression, and found that the Nepali monkeys expressed class I MHC alleles common in animals of Indian origin but absent from their Chinese counterparts.

Keyes's group acknowledges that these results are based on analyses of a small, isolated population of animals, but, as they write in the American Journal of Primatology, "its geographic location within the country and previous opportunities for migration (gene flow) to and from the surrounding areas suggest that this population may be a representative sample of Nepali rhesus macaques."

The use of monkeys from Nepal may help ease the demand for Indian macaques, allowing researchers to continue work originally begun with the Indian monkeys. The use of these animals in research would not affect the naturally occurring populations, because the Wildlife Framing, Breeding and Research Working Policy, enacted by the Nepal government in 2003, mandates that only captive-bred monkeys can be used in research.

Tanja Schub 